\title{
BIOSYNTHESIS OF THE FORMYCIN FAMILY
}

\author{
Takehiko Kunimoto, Tsutomu Sawa, Takashi Wakashiro, \\ Makoto Hori and Hamao Umezawa
}

Institute of Microbial Chemistry, Shinagawa-ku, Tokyo, Japan

(Received for publication January 19, 1971)

\begin{abstract}
It is strongly suggested that none of adenine, adenosine, pool-adenylic acid and RNA-adenine is a direct precursor of formycin while that ribose is exclusively incorporated into the ribosyl moiety. Biosynthesis of formycin does not seem to be closely related to purine metabolism.
\end{abstract}

The formycin family is composed of the C-ribosides of pyrazolopyrimidine derivatives $^{1,2)}$. In a previous paper we proposed a scheme for their biosynthesis in Nocardia interforma as follows: ? $\rightarrow$ formycin B monophosphate $(\mathrm{FMBP}) \rightarrow$ formycin A monophosphate (FMAP) $\rightarrow$ formycin $\mathrm{A}(\mathrm{FMA}) \rightarrow$ formycin $\mathrm{B}(\mathrm{FMB}) \rightarrow$ oxoformycin $\mathrm{B}^{3)}$. This scheme only explains the terminal steps of the biosynthetic pathway while the major part, namely the formation of the C-riboside linkage and that of the pyrazolopyrimidine ring, remains to be elucidated. Another compound containing the Criboside structure is pseudouridine, a component of tRNA. It was reported that pseudouridine is derived from uridine and that the uridine-pseudouridine interconversion takes place through an intramolecular rearrangement in RNA and not principally through a free uracil or diribosyl uracil intermediate ${ }^{4}$. On the other hand, an enzyme which forms pseudouridine monophosphate from uracil and ribose-5-P was isolated from Tetrahymena pyriformis ${ }^{5)}$ and Agrobacterium tumefaciens ${ }^{6}$. It is not known yet how these two findings can be reconciled. The antitumor antibiotic showdomycin also has the C-riboside structure, the biosynthesis of which has not been fully elucidated yet $^{7)}$. Pyrrolopyrimidine ribosides such as tubercidine, toyocamycin and sangivamycin are reported to be derived from adenosine, $\mathrm{N}(7)$ and $\mathrm{C}(8)$ of adenine moiety being eliminated and the remainder subsequently reacting with PRPP to form the pyrrolopyrimidine riboside structure ${ }^{8,9,10)}$. Thus the biosynthetic pathways of these compounds are an extension of the metabolic pathway to purines or pyrimidines. In contrast, the biosynthesis of formycin family seems very unique. In this paper we will present evidence indicating that the biosynthesis of formycin is not closely related to either the de novo or salvage pathway of purine biosynthesis.

\section{Materials and Methods}

\section{Cultivation}

Nocardia interforma was cultured at $27^{\circ} \mathrm{C}$ under reciprocal shaking in SAKAGUCH flasks each containing $50 \mathrm{ml}$ (experiment 1) or $100 \mathrm{ml}$ (other experiments) of a medium consisting of $1.5 \%(\mathrm{~W} / \mathrm{V})$ sucrose, $0.75 \%(\mathrm{~W} / \mathrm{V})$ Polypeptone, $0.75 \%(\mathrm{~W} / \mathrm{V})$ meat extract, and $0.3 \%$ $(\mathrm{W} / \mathrm{V}) \mathrm{NaCl}$. With the exception of experiment 1 , a seed culture was prepared by 2 days of 
shaking, and a $2 \mathrm{ml}$ aliquot was inoculated into each set of 3 flasks containing fresh medium to initiate the cultivation. Each datum is the mean obtained from three flasks.

\section{Isolation Procedures}

(1) RNA-adenine and adenine in the acid-soluble pool (experiments 2 and 3):

Mycelia were separated from the culture fluid by centrifiugation at $1,500 \times g(15 \mathrm{~cm}$ radius $)$ for 10 minutes. After 2 washes with cold physiological saline, the RNA fraction and the acid-soluble fraction were prepared from the mycelia. Each fraction was made $1 \mathrm{~N}$ with $\mathrm{HCl}$ and was heated in a boiling water bath for 1 hour. The solution was chilled and applied to a charcoal column (bed volume $0.5 \mathrm{ml}$ ) which was subsequently washed with water. The column was eluted with isopropanol-conc. $\mathrm{NH}_{4} \mathrm{OH}$ - water $(50: 2: 48, \mathrm{~V} / \mathrm{V})$ and the eluate was evaporated to dryness in vacuo. The residue was dissolved in water and applied to a column of Dowex-1 $\times 2\left(\mathrm{Cl}^{-}\right)(0.8 \times 10 \mathrm{~cm})$. The effluent and water washes were combined, concentrated in vacuo and spotted on Toyo $51 \mathrm{~A}$ paper, which was developed with isoamylalcohol-5\% $\mathrm{Na}_{2} \mathrm{HPO}_{4}(1: 1, \mathrm{~V} / \mathrm{V})$. On the chromatogram, adenine (Rf 0.46) was localized by UV-light, the area was cut out and eluted with water. An aliquot was taken for determination of radioactivity and the amount of adenine in the remaining portion was determined by measuring the optical density at $260 \mathrm{~m} \mu(\varepsilon 13,200)$.

(2) Formycin and adenosine :- In experiment 1, the supernatant of the culture broth was applied to a column of Dowex-50 $\left(\mathrm{H}^{+}\right.$form ; $\left.0.8 \times 10 \mathrm{~cm}\right)$ and the column was washed with water. Formycin was eluted with $50 \mathrm{ml}$ of $0.5 \mathrm{~N} \mathrm{NH}_{4} \mathrm{OH}$. After neutralization, the formycin fraction applied to a column of Dowex-1 $\left(\mathrm{Cl}^{-}\right.$form; $\left.0.8 \times 10 \mathrm{~cm}\right)$. The effluent and water washes were combined and applied to a column of charcoal $(0.8 \times 5 \mathrm{~cm})$, the column was washed with water. Inorganic salts were removed by this procedure. Formycin in the $n$-propanol - conc. $\mathrm{NH}_{4} \mathrm{OH}$ - water $(50: 5: 45)$ eluate was concentrated by evapration in vacuo and submitted to paper electrophoresis under $3,500 \mathrm{~V}$ for 15 minutes in formic acid-acetic acid - water $(25: 75: 900, \mathrm{~V} / \mathrm{V})$. On the paper, formycin was identified by its fluorescence under UV-light and the area was cut out. Formycin was eluted from the paper strip with water. The eluate was made $1.5 \mathrm{~N}$ with $\mathrm{HCl}$ and heated at $100^{\circ} \mathrm{C}$ for 2 hours. Under these conditions adenosine which accompanied formycin was hydrolyzed to adenine, hypoxanthine and ribose while formycin was quantitatively deaminated to formycin B. After neutralization, the hydrolysate was submitted to charcoal column chromatography in a similar manner to that described above. Salts and ribose were removed in the effluent and wash water. After elution from the charcoal column with $n$-propanolconc. $\mathrm{NH}_{4} \mathrm{OH}$ - water $(50: 5: 45)$, formycin $\mathrm{B}$ was separated from adenine and hypoxanthine by paper electrophoresis as abeve. Formycin B thus obtained was more than $95 \%$ pure which was determined by the antibiotic titer (against Xanthomonas oryzae) per unit of UV absorption. Radioactivity of the final preparation of formycin B was multiplied by a factor corresponding to the overall recovery of antibiotic through these procedures. The recoveries of formycin and formycin $B$ were determined on the basis of their antibiotic titers. In other experiments, the fraction containing both formycin and adenosine was prepared from the culture fluid by adsorption and elution from charcoal, column chromato graphy with Dowex-50 and additional column chromatography with Dowex -1 . The datailed procedures were identical to those stated above. The fraction thus obtained was dried in

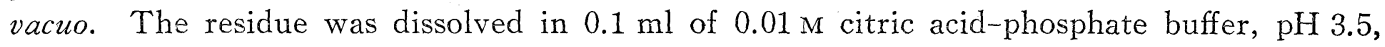
and applied to a column of Sephadex G-10 $(1.25 \times 45 \mathrm{~cm})$ which had been equilibrated with the same buffer solution. Elution was conducted with the same buffer and adenosine and formycin was measured in the eluate by optical density at $260 \mathrm{~m} \mu$ and $295 \mathrm{~m} \mu$ respectively. Formycin was eluted faster than adenosine with excellent scparation. The fractions in each peak were combined and samples were taken for measurement of radioactivity and optical density. The molecular extinction coefficients of adenosine and formycin at $\mathrm{pH} 3.5$ were $14,700(260 \mathrm{~m} \mu)$ and $11,200(295 \mathrm{~m} \mu)$, respectively.

(3) The chromophore fraction:- In experiment 4, each sample of radioactive formycin 
oxidized with periodate ${ }^{11)}$ to give quantitatively 7-amino-pyrazolo[4, 3-d]-pyrimidine-3carboxylic acid (called "chromophore fraction") in crystalline form.

\section{Results}

Incorporation of Various Radioactive Precursors into

Formycin (Experiment 1)

A difficulty which we encountered in the course of this study was that Nocardia interforma, the producer of this antibiotic, did not produce formycin in any chemically defined medium. Several attempts were made to cultivate the cells in the organic medium (see materials and methods) for the first 2 or 3 days and then to transfer the cells to chemically defined media for possible production of formycin, but none was successful. Because of this difficulty, labeling experiments were conducted in the organic medium, and it was impossible to know to what extent an added radioactive precursor was diluted by unlabeled material in the medium. In spite of this risk, we conducted this experiment in the hope that a close precursor would be incorporated into formycin to a significant extent. As shown in Table 1, the incorporation efficiency of all the nucleic acid bases was low, in contrast to that of sugars and some amino acids. This suggests that neither preformed purine nor pyrimidine is a close precursor of formycin. It should be noticed that the incorporation efficiencies of adenine-8- ${ }^{14} \mathrm{C}$ and adenine-

Table 1. Incorporation of various radioactive precursors into formycin

\begin{tabular}{l|c|c}
\hline & $\begin{array}{c}\text { Amount of } \\
\text { radioactivity } \\
\text { added to culture }\end{array}$ & $\begin{array}{c}\text { Percent } \\
\text { incorporation }\end{array}$ \\
\hline Adenine-U-14 C & $14.6 \mu \mathrm{Ci}$ & 0.024 \\
Adenine-8-14 $\mathrm{C}$ & 12.5 & 0.023 \\
Uracil-5- ${ }^{3} \mathrm{H}$ & 21.8 & 0.0035 \\
Orotic acid-6-14C & 3.95 & 0.012 \\
Glucose-U- ${ }^{14} \mathrm{C}$ & 20.0 & 0.22 \\
Ribose-U-14 $\mathrm{C}$ & 9.48 & 0.59 \\
Na-Acetate-2-14C & 3.25 & 0.09 \\
Na-Formate-U-14C & 5.0 & 0.03 \\
Serine-U-14C & 4.21 & 0.25 \\
Glycine-U-14C & 20.0 & 0.26 \\
Aspartic acid-U- ${ }^{14} \mathrm{C}$ & 4.20 & 0.104 \\
Glutamic acid-U-14C & 4.24 & 0.212 \\
\hline
\end{tabular}

Each radioactive precursor was added on the 3 rd day of incubation together with coformycin*, the final concentration of which was made $1 \mu \mathrm{g} / \mathrm{ml}$. The incubation was terminated on the 5 th day when an aliquot of the culture broth was assayed for antibiotic titer (formycin production) while the rest was used for isolation of formycin. The incorporation rate represents :

$$
\frac{\mathrm{dpm} \text { of resulting formycin } \mathrm{B}}{\mathrm{dpm} \text { of respective precursor }} \times 100(\%)
$$

* An inhibitor of adenosine deaminase ${ }^{12}$. $\mathrm{U}-{ }^{14} \mathrm{C}$ were similar. Since $\mathrm{C}(8)$ of adenine cannot be incorporated into pyrazolopyrimidine without being first split off, these incorporation efficiencies are probably the result of incorporation of some fragments of the adenine molecule after degradation.

Time Course Study of Formycin and Cellular RNA-Adenylic

Acid Production by Nocardia interforma (Experiment 2)

The results shown in Table 1 suggested that free adenine was not a direct precursor of formycin. Accordingly, as in the case of the intra-RNA formation of a pseudouridylic acid residue from an uridylic acid residue, the adenylic acid residue in RNA (abbreviation: RNA-adenine) might be a possible precursor of formycin. In Fig. 1, the accumulation of formycin in the culture fluid and of RNA-adenine in the mycelia are plotted against the cultivation period. Both the amount of formycin and of RNA-adenine are expressed as $\mu$ moles per $\mathrm{ml}$ of the whole culture. The accumulation of formycin in the mycelium was practically negligible. Formycin started rising at the 3rd day in contrast to the almost stationary level of RNA-adenine during the 
Fig. 1. The time course of formycin production and of the amount of adenylic acid residue in the cellular RNA.

The amount of formycin was determined on the basis of antibiotic titer. RNA-Adenine was isolated and determined as stated in methods.

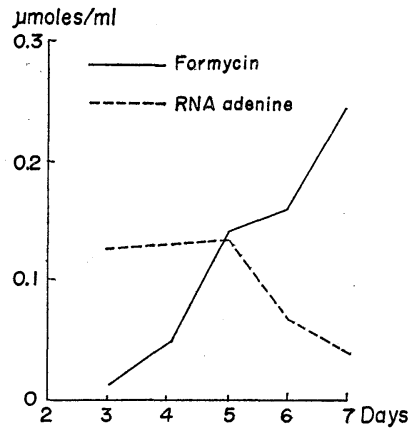

Fig. 2. Total and specific radioactivities of formycin, adenosine, adenine nucleotides and RNA-adenine isolated from a culture labeled with adenine-U-14 C.

(a). Adenine-U-1 ${ }^{4} \mathrm{C}(0.25 \mu \mathrm{Ci})$ was added to each of 3 cultures at 48 -hour of incubation. At 1 day intervals afterwards, $10 \mathrm{ml}$ aliquots were drawn from each culture and 3 samples were combined and stored in a refrigerator until they were submitted to the isolation procedures.

(b). Adenine in the acid-soluble fraction of cells is arbitrarily expressed as adenine nucleotides.
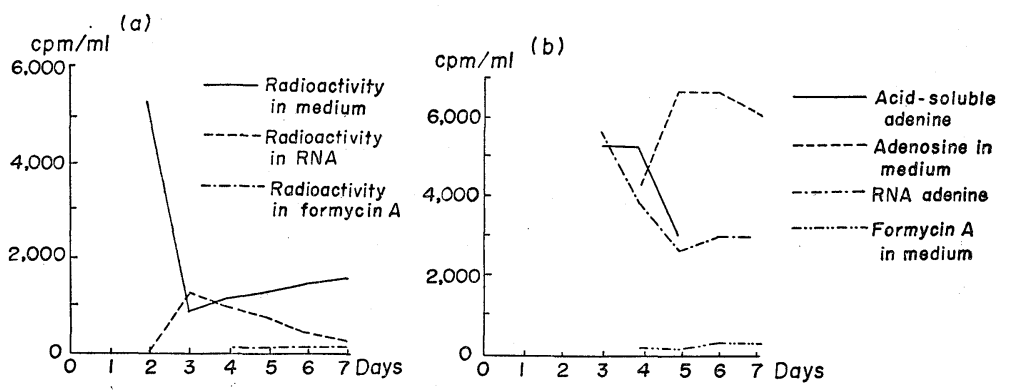

3rd to 5 th day of culture. From the 5 th to 7 th day, the accumulation of formycin seems to be stoichiometrically accounted for by the decrease in RNA-adenine content. In consideration of possible turnover of RNA-adenine, especially in the earlier period of culture, RNA-adenine might be considered as a precursor of formycin. In order to clarify this and other equivocal points, the following experiments were conducted.

Comparison of the Specific Radioactivity of Formycin with those of

Extracellular Adenosine, Pool Adenylic Acids and RNA-Adenine

Isolated from a Culture Labeled with ${ }^{14} \mathrm{C}-\mathrm{U}$-Adenine (Experiment 3 )

$\mathrm{U}-{ }^{14} \mathrm{C}$-Adenine was added to a 2-day culture of Nocardia interforma. At intervals thereafter, an aliquot of the culture (mycelial suspension) was withdrawn for the determination of radioactivity and specific radioactivity in appropriate fractions. As shown in Fig. 2-a, radioactive adenine was quickly taken up into cells and a part of the radioactivity was found in the mycelial RNA fraction. Most of the remainder was thought to be in the cellular acid-soluble pool, DNA was also presumably labeled to some extent. The radioactivity in the formycin fraction remained very small. In order to show the transition of radioactivity in more stoichiometrical way, radioactivities of several metabolites including formycin were expressed as 'cpm per umole', or 'specific radioactivity', as shown in Fig. 2-b. It is apparent from the result that adenine is efficiently incorporated into extracellular adenosine, pool adenylic acids and RNA-adenine but not into formycin. The specific radioactivities of the normal metabolites are 10 30 times higher than that of formycin throughout the cultivation. In a separate experiment, it was found that the specific activity of RNA-guanine was similar to that of RNA-adenine. This finding is a good evidence that adenine, adenosine, adenine nuleotides or RNA-adenine (or guanine) is not a precursor of formycin.

\section{Origin of the Ribose Moiety (Experiment 4)}

As seen above, the biosynthetic route to formycin seems unique and different from those of purine nucleoside antibiotics and of pyrrolopyrimidine antibiotics. A 
study was made to determine the origin of the ribose moiety of formycin. Since formycin is a very stable compound, there are only a few ways to chemically decompose the molecule. Periodate oxidation, which is schematically shown in Table 2 , is one such chemiacl reaction ${ }^{11}$. Radioactive preparations of formycin made from U-14 $\mathrm{C}$-ribose, U- ${ }^{14} \mathrm{C}$-glucose, U${ }^{14} \mathrm{C}$-aspartic acid, U- ${ }^{14} \mathrm{C}$-glycine or $\mathrm{U}-{ }^{14} \mathrm{C}$-glutamic acid were oxidized and the chromophore fraction of each was isolated. For each sample, the specific radioactivity of formycin (I) and that
Table 2. Origin of the ribose moiety

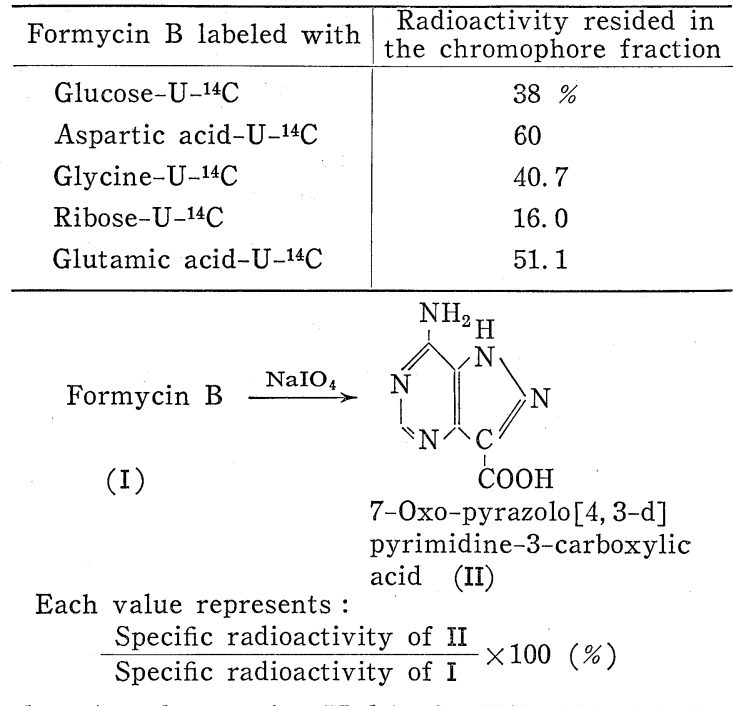
of the chromophore (II) were determined. As shown in Table 2, II/I $\times 100(\%)$ for formycin labeled from $\mathrm{U}-{ }^{14} \mathrm{C}$-ribose was $16 \%$ which is significantly low compared to the other values. The result could be interpreted as follows: $\mathrm{U}-{ }^{14} \mathrm{C}-$-ribose had been incorporated solely into the ribose moiety of formycin and consequently the chromophore fraction derived from it retained only one ${ }^{14} \mathrm{G}$ atom out of 5 of the original ribose moiety.

\section{Discussion}

Our previous proposal for the biosynthesis of the formycin family was: ? $\rightarrow$ FMBP $\rightarrow$ FMAP $\rightarrow$ FMA $\rightarrow$ FMB $\rightarrow$ oxoformycin ${ }^{3)}$. An important question is the mechanism of formation of formycin B-P. According to the data described in this paper, ribose appears to be a direct precursor of the ribosyl moiety of formycin. Therefore, we suggest that the ribosyl moiety binds to a precursor of the pyrazolopyrimidine ring and that this putative intermediate is phosphorylated at $5^{\prime}$ of the ribosyl moiety. If this were not the case, we would expect the riboside (formycin B) to diffuse out of the cells as soon as the chromophore moiety is completed. Formycin B has never been detected in the culture fluid before formycin accumulates. Formation of formycin by extension of de novo purine nucleotide synthesis is also unlikely because under the conditions where only $0.25 \%$ of the radioactivity given as $\mathrm{U}^{14} \mathrm{C}-$ glycine was recovered in formycin (Table 1 ), about 10 times as much radioactivity was distributed in adenosine (the result is not given). Thus the major part of formycin biosynthesis does not seem to be related to purine biosynthesis.

\section{References}

1) Koyama, G.; K. Maeda, H. Umezawa \& Y. Irtaka: The structural studies of formycin and formycin B. Tetrahedron Letters 1966-6: 597 602, 1966

2) Koyama, G. : On the chemistry of formycins. Ph. D. Thesis, Tokyo University, 1969

3) Sawa, T.; Y. Fukagawa, I. Homma, T. Wakashiro, T. Takeuchi, M. Hori \& T. Komai : Metabolic conversion of formycin B to formycin A and oxoformycin B in Nocardia interforma. J. Antibiotics $21: 334 \sim 339,1968$

4) Ginsberg, T. \& F. F. Davis : The biosynthesis of pseudouridine in ribonucleic acids of Escherichia coli. J. Biol. Chem. $243: 6300 \sim 6305,1968$

5) Heinrikson, R. L. \& E. Goldwasser : Studies on the biosynthesis of 5-ribosy1 5'-monophosphate in Tetrahymena pyriformis. J. Biol. Chem. $239: 1177 \sim 1187,1964$ 
6) Suzuki, T. \& R. M. Hochster : On the biosynthesis of pseudouridine and of pseudouridylic acid in Agrobacterium tumefaciens. Canad. J. Biochem. 44:259 272, 1966

7) Suhadolnik, R. J. \& R. M. Ramer : 156th Nat1. Meeting American Chem. Soc. Abstract 26, 1968

8) Smulson, M. E. \& R. J. Suhadolnik : The biosynthesis of the 7-deazaadenosine ribonucleoside, tubercidine, by Streptomyces tubercidicus. J. Biol. Chem. $242: 2872 \sim 2876,1967$

9) Uematsu, T. \& R. J. Suhadolnik : Nucleoside antibiotics. VI. Biosynthesis of the pyrrolopyrimidine nucleoside antibiotic toyocamycin by Streptomyces rimosus. Biochemistry $9: 1260 \sim$ 1266, 1970

10) Suhadolnik, R. J. \& T. Uematsu : Biosynthesis of the pyrrolopyrimidine nucleoside antibiotic, toyocamycin. VII. Origin of the pyrrole carbons and the cyano carbon. J. Biol. Chem. 245 : 4365 4371, 1970

11) Kawamura, K.; S. Fukatsu, M. Murase, G. Koyama, K. Maeda \& H. Umezawa: The studies on the degradation products of formycin and formycin B. J. Antibiotics, Ser. A $19: 91 \sim 92,1966$

12) Sawa, T.; Y. Fukagawa. I. Homma, T. Takeuchi \& H. Umezawa : Formycin-deaminating activity of microorganisms. J. Antibiotics, Ser. A $20: 317 \sim 321,1967$ 